\title{
Research on the Interactive Design of Electronic Magazine in the New
}

\section{Media Era}

\author{
HuiHuang ${ }^{1, a}$, Junjian Liu $^{2, b}$ Jianchun Shao ${ }^{3, c}$ \\ ${ }^{1}$ School of Art and Design, Jingdezhen Ceramic Institute, Jingdezhen, 333004, China \\ ${ }^{2}$ Jingdezhen Ceramic Vocational Technical College, Jingdezhen, 333400, China \\ ${ }^{3}$ Jingdezhen Ceramic Vocational Technical College, Jingdezhen, 333400, China \\ a114249216@qq.com, ${ }^{\mathrm{b}} 48858523 @ q q . c o m,{ }^{\mathrm{c} j d z s j c @ 163 . c o m}$
}

Key words: electronic magazine; interaction; interactive design

Abstract. The interactivity is a major characteristic of interactive design of electronic magazine in the new media era. The electronic magazine includes seven interactive elements: icon, navigation, switching, rolling, link, index, comment and interaction. Besides, it has three interactive design methods: interactive parsimony, specialized service and joyful experience.

\section{Introduction}

The interactivity is a major characteristic of interactive design of electronic magazine in the new media era, which is also an advantage differentiating electronic magazine from traditional magazine. The process of interaction involves two aspects: one is the information transmission of content, and the other is the readers' reception and feedback. During this process, readers are able to understand and grasp any information actively.

\section{The interactive elements of electronic magazine}

Interactivity is one of the advantages of electronic magazine, which enables readers to participate, and changes the traditional single mode of transmission, so that the information can be spread more broadly. The feature of interactivity brought readers an unprecedented audio-visual experience. It enables readers to read selectively and occupy a dominant position, and gets them involved, which also reflects the user-centered and humanized design of electronic magazine.

Icon. Icon is a principal element indispensable in interactive design, which plays the role of instructing. It not only guides the interaction between readers and electronic magazine but also serves as a bridge linking the readers and electronic magazine, thus deepening readers' understanding of electronic magazine. Emerging in the form of LOGO, the icon has become the leading mark and control key of reading the electronic magazine.

Navigation. Navigation is just like the road guiding the readers to know electronic magazine in interactive design. If the icon is an arrow, then the navigation is the map. The navigation in the electronic magazine has the page of operational description and active directory. The directory can either be opened or hidden, visually showing the framework of the magazine, so that readers can select their interested chapters and sections at a glance. The directory navigation allows readers to control and select the desired inside pages freely. Many directories arr designed in such a way that when people roll mouse over or the fingers touch the title, there will be a pop-up page, which contains simple introductory texts, pictures, animation, etc., facilitating readers to better choose when reading. 
Switching. The vast majority of interactions in electronic magazine are achieved through switching. For example, the opening and closing of pictures, and the opening and hiding of tips, which improves the mystery of electronic magazine and the interest when readers experience the electronic magazine. The switching of pictures can be achieved in different ways, such as smooth rolling, cube, horizontal switching and vertical switching. During the process of making electronic magazine, the designers can choose the speed and time interval of picture switching as well as the switching mode. Different switching combinations pose lots of novelties and challenges to designers in the process of making electronic magazine, and gives readers a sense of freshness and interest in reading.

Scrolling. The interactive scrolling of electronic magazine is mainly manifested in two aspects of text and spread. Because of the limitation of the size and resolution of the iPad screen, the text typography can't adjust its size and spacing optionally as the print magazines, which causes that the body text can be presented to readers only by scrolling. The text scrolling is divided into horizontal scrolling and vertical scrolling, and for the convenience of page turning, vertical scrolling is generally adopted to handle the text layout. Besides, the text scrolling can also be divided into full text scrolling and partial text scrolling. And different scrolling settings give readers different experience effects. The spread scrolling is a major feature of the interactive design of electronic magazine. Because of the single-screen display of iPad, the large spread pictures need the guidance of spread icon, so that readers can watch in a scrolling way. For the overall display of the picture, readers can click on the picture to see the full large picture.

Link. The electronic magazine itself exists in the form of hypertext links. Link is not only another feature of the interaction elements of electronic magazine, but also an independent function higher than print magazine. It allows readers to click on and browse the relevant articles or websites in the network environment, thus enabling them to better understand other relevant information about the magazine content. The link has the effect of drawing inferences about other cases from one instance, and the function of database. The electronic magazine not only contains the internal links of secondary reports and the full text but also contains many external links, such as links with other electronic magazines and the database of bibliographic services.

Index. The index mainly refers to the specific reading mode provided to mobile phone users in the process of making electronic magazine, so that they can read on the platform of iPad and mobile simultaneously. In the process of making electronic magazine, the title, image, text and other contents should be indexed and sorted, so the mobile phone users are able to read the article according to the order. This is not only an example of cross-platform design, but also a survival skill to adapt to the whole media in the development of electronic magazine.

Comment and interaction. The interactivity of electronic magazine includes human-computer interaction, human-content interaction and human-human interaction. Only the diversified interactive methods and features can highlight the interactive advantages of electronic magazine. Readers can comment online in real time on the magazine content, the magazine layout and the operation convenience when reading electronic magazine. At the same time, they can communicate with users who are also reading the magazine online. Therefore, the readers' comments and feedback can be presented to the public without the limitation of time and space. Except the person in charge of the online magazine, any user can exchange his comments.

\section{The interactive design method of electronic magazine}

The super strong interactivity is one of the prominent advantages of electronic magazine. The network and computer multimedia technology enable the interactive function of electronic 
magazine to be realized. The interaction can give readers more information in the process of reading, achieving the extension of information, and readers can also feel relaxed and enjoyable like playing games in the process. The interaction changes the passive readers into active ones, which is the core value of the development of electronic magazine. The interactive design of electronic magazine also has certain design methods.

Interactive parsimony . Because the elements of electronic magazine are very rich, integrating the image, text, video, animation and audio together, so in the electronic interactive design of visual and interactive elements, it is important to use the method that simplifies the operation. The layout of electronic magazine has a large amount of information which is progressively layered, so if the readers want to read all the wonderful and unpredictable contents of the magazine, they must complete with their own operation. The operation of readers is like a open door and the interactive icon is the key to opening the gate of rich contents. People can enjoy the obscure, wonderful and striking scenery only when the gate is easy to be opened.

Therefore, the excellent electronic magazine must be the unified whole of simple operating mode and complex structure. Meanwhile, the designers should take into account the influence of age, gender, occupation and cultural degree on the receptivity of interactive design of magazine. Besides, they should carry out specific design based on the specific circumstances of the magazine readership.

The specialized service. Currently, there are all kinds of magazines on various major electronic magazine platforms. The aim of any magazine brand is to make its own magazine unique, with distinct personality and style. To design the electronic magazine with unique characteristics, in addition to the fineness of content and visual design, it can also resort to the interactive technology, thus making the magazine which is targeted and service-oriented.For example, the electronic magazine AutoWeek is an introductory magazine about the latest auto types and test drive experiences. The electronic magazine has comprised many targeted interactive elements, such as the button of viewing the enlarged drawing of certain parts of the car, enabling consumers to better understand the detail advantage of the car. Besides, it has provided the relevant links of the part, so that readers can make a detailed comparison and have a deeper understanding of products of this type.

The joyful experience. The design stresses the service concept of "putting people first" and "taking the users as the center", whose essence is to make sure that the users' experience is pleasant and comfortable. The development and progress from the traditional paper magazine design to the interactive design of electronic magazine is constantly improving its service function, so as to facilitate the reading. The purpose of the design is to present the content to the user more clearly and service them better. Therefore, the design pays much attention to humanization.

The humanization of the interactive design of electronic magazine is also reflected in that it gives readers the supreme choice. For example, in the use of interactive control, readers can choose to either click on the extended reading or just browse the main content without reading the nearby information; in the use of background music and video, it also gives readers corresponding option, and provides different audios and videos for readers to choose. It gives high priority to reader's choices and pays attention to the reader's preferences. The joyful experience is not only the prerequisite for the survival of the magazine but also one of the factors to attract readers.

\section{Summary}

In all the excellent electronic magazines, the interactive elements are highly unified with the content and style of the magazines, which adds the finishing touch to show the charm of the magazines. As 
to the "quantity" of interactive elements design, on the one hand, the interactive elements are indispensable, but on the other hand, they can not be too many. The lack of interactive element will lose its nature of interaction or make it unable to be called as the multimedia interactive electronic magazine; however, if there are too many interactive elements, it will be inevitably messy, which affects the reading of magazines.

\section{References}

[1]ZhenYa, Preliminary study on Interaction Design, The Science Education Article Collects,2011 , (12):62, 92-95

[2]LiShanshan, The application research of the electronic magazine layout design, 2008

[3]TongXianwen,Analysis of the design of network electronic magazine,YiHai,2011 , (10):59 